Probability and Typicality in Deterministic Physics

Meir Hemmo ${ }^{1}$ and Orly Shenker ${ }^{2}$

\begin{abstract}
Recently it has been argued that typicality considerations play a crucial explanatory role in deterministic theories in physics (e.g. classical statistical mechanics and Bohmian mechanics). In this approach a sharp distinction is made between typicality and probability. We analyze in this paper the relation between the notion of typicality and probability, the question of the choice of measure in deterministic theories in physics, and the way in which probability and typicality arise and should be understood in such theories. We argue that in deterministic theories it is the notion of probability rather than typicality that may (sometimes) have explanatory value.
\end{abstract}

Acknowledgement: This research is supported by the Israel Academy of Science, grant number $713 / 10$.

\footnotetext{
1 Philosophy Department, University of Haifa, Haifa 31905, Israel. meir@research.haifa.ac.il

2 The Hebrew University of Jerusalem, Jerusalem 91905, Israel. orlyshenker@pluto.mscc.huji.ac.il.
} 


\section{Probability and Typicality in Deterministic Physics}

\section{Introduction: The Typicality Approach}

This paper analyses the meaning of typicality considerations in physics, and their relation to the notion of probability. The structure of the paper is as follows. We begin in this section by describing the so-called typicality approach (as framed in the context of deterministic theories in physics, e.g. classical statistical mechanics, Bohmain mechanics). In section 2 we describe the way in which probabilistic statements in classical statistical mechanics ought to be understood. In section 3 we examine arguments based on the classical dynamics to the effect that the Lebesgue measure is natural in statistical mechanics. In section 4 we analyze the significance of Lanford's theorem in classical statistical mechanics, and we explain how the theorem ought to be understood without appealing to typicality. In section 6 we consider the typicality argument in Bohmian mechanics. Section 7 presents an open question for future research concerning indeterministic (stochastic) theories.

In classical statistical mechanics the standard way of understanding the thermodynamic behavior of systems around us appeals to a probability distribution over the initial microstates of the systems (compatible with the initial thermodynamic macrostate). On the standard way of thinking one says that given the uniform probability distribution (relative to the Lebesgue measure) over the initial macrostate, it is highly probable that the system will, for example, approach equilibrium after some designated time. In this way, the behavior of the system is explained by the fact that its actual microstate is highly likely to sit on a trajectory, which will take it to equilibrium at the time in question. Here the high probability pertains to subsystems of the universe, and it is assumed further that the trajectory of the whole universe that gives rise to this high probability itself sits on an initial condition which has high probability. Note that here there are two notions of probability: a probability distribution over the initial macrostate (i.e. the microstates compatible with the macrostate at some present time) of subsystems of the universe, and a probability distribution over the initial conditions of the universe.

Another important example of the central role played by the measure in explaining physical behavior in statistical mechanics is in Einstein's $(1905,1926)$ account of 
Brownian motion, as developed by Wiener (see Pitowsky 1992). As is well known, Wiener has proved that the so-called Wiener measure of trajectories in the phase space of a Brownian particle which are continuous but nowhere differentiable is one. The explanation of the actual behavior of Brownian particles is based on the assumption that their actual trajectories belong to this measure one set. Avogadro's number is derived from this assumption.

A question that immediately arises concerning this understanding is what could a probability distribution over the initial conditions of the universe possibly mean. A probability distribution suggests some sort of a random sampling of an initial condition out of the set of all possible conditions. But with respect to the initial conditions of the universe any such sampling (if it is to be physical) would be external (and prior) to the universe, and therefore this seems to suggest an empirically meaningless fairy tale. This problem does not arise with respect to subsystems of the universe, since one can ground a probability distribution over initial conditions in experience (as we show in Section 3). Moreover, probability in physical theories is usually conceived as involving (or as being tested by) repetitions of experiments, which in the case of the initial conditions of the universe are trivially impossible.

We understand the typicality approach ${ }^{3}$ as an attempt to solve these problems by appealing to a certain natural measure over initial conditions, where the measure is not understood as a probability measure (see Maudlin 2007 for a similar construal).

Here is an example of how the distinction between typicality and a probability distribution over initial conditions is made:

"When employing the method of appeal to typicality, one usually uses the language of probability theory. When we do so we do not mean to imply that any of the objects considered is random in reality. What we mean is that certain sets (of wave functions, of orthonormal bases, etc.) have certain sizes (e.g., close to one) in terms of certain natural measures of size. That is, we describe the behavior that is typical of wave

\footnotetext{
${ }^{3}$ For various formulations and extensive discussions of the typicality approach, see Dürr, Goldstein and Zanghi (1992), Maudlin (2007), Callender (2007).
} 
functions, orthonormal bases, etc.. However, since the mathematics is equivalent to that of probability theory, it is convenient to adopt that language. For this reason, we do not mean, when using a normalized measure $\mu$, to make an "assumption of a priori probabilities," even if we use the word "probability." Rather, we have in mind that, if a condition is true of most $D$, or most $H$, this fact may suggest that the condition is also true of a concrete given system, unless we have reasons to expect otherwise." (Goldstein, Lebowitz, Mastrodonato, Tumulka and Zanghi, 2010a).

And in another place (2010b), they say:

"When we express that something is true for most $\mathrm{H}$ or most $\psi$ relative to some normalized measure $\mu$, it is often convenient to use the language of probability theory and speak of a random $H$ or $\psi$ chosen with distribution $\mu$. However, by this we do not mean to imply that the actual $H$ or $\psi$ in a concrete physical situation is random, nor that one would obtain, in repetitions of the experiment or in a class of similar experiments, different $H$ 's or $\psi$ 's whose empirical distribution is close to $\mu$. That would be a misinterpretation of the measure $\mu$, one that suggests the question whether perhaps the actual distribution in reality could be non-uniform. This question misses the point, as there need not be any actual distribution in reality. Rather, Theorem 1 means that the set of "bad" Hamiltonians has very small measure $\mu$. "

There are three different statements made here about the idea of typicality:

(i) The set of initial conditions compatible with the initial macrostate of the universe is divided into two subsets, T1 and T2 such that all the microstates in T1 but not in T2 give rise to some property $\mathrm{F}$. The property $\mathrm{F}$ may be for example the approach to equilibrium in statistical mechanics, or the Born rule in Bohmian mechanics.

(ii) There is some natural (normalized) measure $\mu$ over the initial conditions such that $\mu(\mathrm{T} 1)$ is close to one (and $\mu(\mathrm{T} 2)$ is close to zero). In this sense, most initial conditions, as determined by $\mu$, are in T1 (and are called typical).

(iii) In a given experiment, the actual initial microstate of the universe belongs to T1. 
Let us explain these three statements in turn. The statement in (i) above expresses a contingent fact about the dynamics, namely a fact about how the initial conditions are mapped by the equations of motion into microstates at later times. There are theorems in both statistical mechanics and Bohmian mechanics that demonstrate that various special cases of (i) hold under some conditions, with some appropriate property F. Examples in classical statistical mechanics are Lanford's theorem in which $\mathrm{F}$ is (roughly) entropy increase and the Birkhoff-von Neumann theorem in which $\mathrm{F}$ is the so-called pointwise ergodic theorem. In Bohmian mechanics a special case of (i) is the theorem by Dürr, Goldstein, and Zanghi (DGZ, 1992) in which F is the Born rule (the absolute square of the quantum mechanical wavefunction) which we discuss below. Statement (i) is not controversial in our discussion.

The notion of most in statement (ii) above requires a measure over the phase space. That is, there are infinitely many ways to determine the size of subsets of a continuous set of points. The question is on what grounds one can justify the choice of measure, or the choice of some class of measures. Usually, in classical statistical mechanics the measure chosen is the Lebesgue measure (or the class of measures absolutely continuous with the Lebesgue measure), and in quantum mechanics the measure is given by the absolute square of the wavefunction. The grounds for these choices are that each of these measures has a preferred dynamical status in the theory.

Statement (iii), as stated above, seems as expressing the brute fact, without further reasoning, that the microstate of the universe invariably (in every experimental set up) belongs to T1. But since there are microstates of the universe that don't belong to T1 this fact calls for a justification. It is evident that (ii) is taken in the typicality approach to completely justify (iii), that is if T1 were to contain only a small fraction of the microstates of the universe, one would not see (iii) as justified. It is important to stress that in this approach the justification of (iii) makes no appeal to probability. Rather, it is the measure of T1 that is supposed to do the whole work. This implies that, lacking reasons to expect otherwise, microstates of the universe that belong to T2 are not realized. 
In short, there are two questions that need be answered in the context of typicality: what justifies the choice of measure in (ii), and what justifies the passage from (ii) to (iii). In particular, the question we consider is whether there are grounds that justify the choice of measures in a way that explains the observed behavior of physical systems. If such grounds could be spelled out the problems concerning the meaning of probability distributions over the initial conditions of the universe would obviously evaporate together with the probability distribution itself. In the subsequent sections we attempt to answer these two questions. We will see that statements (ii) and (iii) are both wanting. Again, statement (i) is not controversial in the context of typicality. Our analysis will lead us to reject the typicality approach.

\section{Probability and the Choice of Measure}

In order to set the stage we need to go into some detail concerning the way in which probability statements arise in deterministic theories in physics and how precisely the choice of measure over the state space is carried out. We first focus on classical statistical mechanics.

Consider the paradigmatic case of an ideal gas $S$, which is initially confined by a partition to the left half of a container, and then, by removing the partition, is allowed to expand, and finally fills out the entire container. Suppose that we set up a very large number of such gases $S_{1 \ldots} S_{k}$, all of which are prepared in the same initial macrostate $M_{0}$ in which the gas is confined to the left half of the container by a partition. We then remove the partitions and follow the spontaneous macroscopic evolution of these gases for a certain time interval $\Delta t$, and we see by simple counting that the overwhelming majority of the gases $S_{1 \ldots} S_{k}$ quickly reach and then remain in macrostate $M_{l}$ in which they fill up the entire container. We now wish to predict the evolution of another system, call it $S_{k+1}$, which is prepared in the same initial macrostate as $S_{1} \ldots S_{k}$. We know that the dynamical equation of motion that governs the evolution of $S_{k+1}$ is the same as the ones governing $S_{1} \ldots S_{k}$, but we do not know the details of this dynamics, nor do we know the exact initial microscopic conditions of $S_{k+1}$ and therefore all we can rely on in this prediction is the above experiment.

Can we infer from the experiment with $S_{1} \ldots S_{k}$ that $S_{k+1}$ is highly likely to end up in macrostate $M_{l}$ ? That is, can we use the experiment with $S_{l} \ldots S_{k}$ in order to come up 
with a probabilistic law, on which we can base our bets regarding the evolution of $S_{k+1}$ ? The answer is, of course, yes, we can infer the probabilities from the finite observed relative frequencies. ${ }^{4}$ This inference is valid just to the extent that we can infer from experience any other physical law or prediction, such as $F=m a$. However, the way in which our probabilistic predictions can be justified, and the extent to which they can be justified - are not always clear in the literature, as we show later.

To see how to understand probabilistic statements in statistical mechanics let us describe the above experiment in the phase space of the gas. Classical mechanics tells us that the universe consists of microscopic particles, and that our experience is an effect of the microstate of the universe, which is the state of those particles. However, it is a physical fact that our senses are too coarse to reflect the full details of the microscopic structure of the universe; we can only perceive some of its general features. In this sense our experience is macroscopic. In the above experiment, we can only observe relative frequencies of transitions between macrostates of the gas. Let us see how these transitions are described in the phase space, and then how these relative frequencies are accounted for in the phase space.

The phase space of a system (in our example, of any of the systems $S_{i}$ ) is partitioned into sets of microstates, which are indistinguishable by an observer; these sets are called macrostates. The phase space regions corresponding to the macrostates express the observer's maximal observational capability, and therefore while the observer can tell which macrostate contains the actual microstate of the system at the time of observation, it cannot tell which part of the macrostate contains that microstate.

We now formulate what we take to be the essential way for calculating transition probabilities in statistical mechanics. Suppose that at time $t_{0}$ an observer $O$ finds the system $S$ in macrostate $M_{0}$ (as for example in our experiment above; see Figure 1). Suppose also that $\mathrm{O}$ knows the laws of classical mechanics, which govern S's evolution in time. If $\mathrm{O}$ knows the Hamiltonian of $S$, that is: if $\mathrm{O}$ knows the equations of motion of $S$, then $\mathrm{O}$ can (in principle) calculate the evolution of all the trajectory segments that start out in the microstates contained in $M_{0}$ and find out the end points

\footnotetext{
${ }^{4}$ This inference is a subtle issue which depends on how probability is understood. We don't address this question here.
} 
of these trajectory segments after the time interval $\Delta t$. These end points make up a set, which we call the dynamical blob $B\left(t_{0}+\Delta t\right)$ of $S$ at $t_{0}+\Delta t$ given that it was in $M_{0}$ at $t_{0}$. In general, the region covered by $B\left(t_{0}+\Delta t\right)$ overlaps with several macrostate regions; for instance, it may partially overlap with $M_{l}$ (in which the gas fills our the entire container), and with some other macrostates, such as $M_{2}$ or $M_{3}$ in which the macrostate of the gas is different. If the system $S$, which started out in $M_{0}$ at $t_{0}$, is observed to be (say) in macrostate $M_{l}$ at $t_{0}+\Delta t$, then this means that the microstate of $S$ is actually in the region of overlap between the region of macrostate $M_{l}$ and the region of the dynamical blob $B\left(t_{0}+\Delta t\right)$. Now, in our above experiment, $\mathrm{O}$ carries out the experiment $k$ times (or on $k$ identical systems). In some of these experiments actually in most of them (in our story) - at $t_{0}+\Delta t$ the system $S$ is observed to be in $M_{l}$ and in other fewer experiments it is found in $M_{2}$ or $M_{3}$, or more precisely in the regions of overlap of the dynamical blob $B\left(t_{0}+\Delta t\right)$ with these macrostates, with some relative frequencies $F_{1}, F_{2}$ and $F_{3}$ respectively. These relative frequencies are the empirical basis on which the probabilistic statements of the theory can be based, and on the basis of which these statements can be tested or justified.

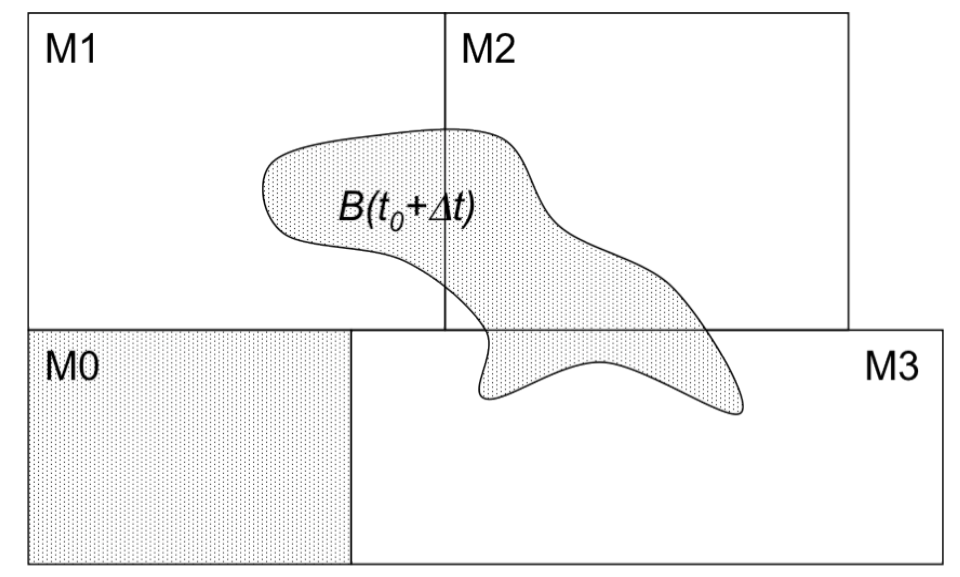

Figure 1

The next step towards constructing or justifying the probabilistic theory is as follows. Given the above experimental outcomes, we have the relative frequencies with which systems of type $S$ that start out in $M_{0}$ at $t_{0}$ are found in the macrostates $M_{1}, M_{2}$ or $M_{3}$. We conclude that the phase points of our $k$ systems evolved into the regions of overlap of the dynamical blob $B\left(t_{0}+\Delta t\right)$ with the macrostates $M_{1}, M_{2}$ or $M_{3}$. We then conjecture on the basis of our experience that this statistical behavior will be repeated 
(more or less) in the future. Since any of the microstates in $M_{0}$ is a possible initial condition of $S_{k+1}$ and since the phase space is continuous, such a generalization of our experience requires that we impose a measure on the phase space. We identify the set of probability measures that, if applied to the continuous phase space of $S$, yield a measure of the regions of overlap of the blob $B\left(t_{0}+\Delta t\right)$ with the macrostates $M_{1}, M_{2}$ or $M_{3}$ that are (to a satisfactory approximation) identical with the relative frequencies $F_{1}$, $F_{2}$ and $F_{3}$, respectively. There are many - possibly infinitely many - such measures, and all of them are empirically adequate. Among them we choose one measure, using pragmatic criteria such as simplicity, convenience, meshing with other theories, etc.. Call this measure $\mu$. The (normalized) measures of the regions of overlap are then given by $\mu\left(B(t) \cap M_{1}\right) \approx F_{1}, \mu\left(B(t) \cap M_{2}\right) \approx F_{2}, \mu\left(B(t) \cap M_{3}\right) \approx F_{3}$. This measure $\mu$ is imposed over the blob $B\left(t_{0}+\Delta t\right)$ and provides the basis for predicting the evolution of system $S_{k+1}$ in terms of transition probability (roughly) as follows:

(*) The transition probability that $S_{k+1}$ will evolve to macrostate $M_{i}$ at $t_{0}+\Delta t$ given that it was in macrostate $M_{0}$ at $t_{0}$, is equal to

$$
\mu\left(B\left(t_{0}+\Delta t\right) \cap M_{i} \mid B\left(t_{0}\right)=M_{0}\right) \approx F_{i} .
$$

That is, the transition probability from the macrostate $M_{0}$ at $t_{0}$ to $M_{i}$ at $t_{0}+\Delta t$ is equal to the (normalized) measure of the region of overlap of the blob $B\left(t_{0}+\Delta t\right)$ with the macrostates $M_{i}$. This is the basis of our probabilistic theory.

Note that in general $\mu\left(M_{i}\right) / \mu\left(M_{j}\right)$ need not be equal to $\mu\left(B(t) \cap M_{i}\right) / \mu\left(B(t) \cap M_{j}\right){ }^{5}$ Note further that despite the deterministic dynamics these transition probabilities between macrostates are physically objective provided the partition to the macrostates is objective. ${ }^{6}$

What is the significance of taking the $\mu$ (normalized) measure over the blob $B\left(t_{0}+\Delta t\right)$ as underwriting our probabilities for measurement outcomes? It is crucial to see that the probabilistic statements are about transitions from $M_{0}$ at $\mathrm{t}_{0}$ to any one of the

\footnotetext{
${ }^{5}$ This implies that the transition to a given macrostate need not be equal to the entropy of that macrostate, even if both are measured by the same measure $\mu$.

6 This last condition needs to be flashed out; we skip this here.
} 
macrostates $M_{i}$. We don't distribute probabilities relative to the $\mu$ measure over the initial macrostate $M_{0}$ at $t_{0}$. Of course, if the measure $\mu$ is invariant under the classical dynamics, e.g. if it happens to be the Lebesgue measure, then one can map, backwards (as it were), the measure of regions over the blob at later times to the corresponding regions over the initial mactostate. That is, in this case the measure of a set of points in $M_{0}$ is equal to the measure of the time evolved set of points to which it is mapped by the dynamics. Once the (normalized) measure is fixed (by the probabilities) one can distribute uniform probabilities relative to the Lebesgue measure over the initial macrostate. But note that this interpretative move is derivative. In general, whether or not the measure that best fits our observations is the Lebesgue measure, or more generally a measure that is invariant under the dynamics, is a contingent matter.

We can now see what justifies the choice of measure and what justifies probabilistic statements in classical statistical mechanics, and moreover how these two issues are related. First, probabilistic statements are grounded in the experience of relative frequencies in the way stated above. Second, the choice of measure is dictated inductively (not uniquely) by the observed relative frequencies. That is, the measure is implied by the probabilities rather than the other way around. We can only justify empirically transition probabilities as sketched in $(*)$ above rather than distributions over initial conditions.

The implications of this analysis for the typicality approach are as follows.

(I) The probability measure $\mu$ is applicable only to subsystems of the universe. Of course, if the dynamics is deterministic, each microstate of all the subsystems of the universe can be mapped backwards to the initial conditions and the measure over the initial conditions will depend on the measure at the later times. But in this way the justification of the choice of the measure over the initial conditions is grounded in experience, and therefore it cannot be taken to explain (non-circularly) experience. Note that this argument applies to the question of the choice of the measure regardless of whether the measure is understood as determining the typical set of initial 
conditions (as in the typicality approach) or as a probability measure over the initial conditions of the universe (as in standard approaches to statistical mechanics).

(II) This strategy of grounding the measure over the initial conditions of the universe in experience can hold only with respect to a fraction of all possible initial conditions of the universe (compatible with the initial macrostate). It excludes by construction initial conditions that lead to a universe at the later times which is macroscopically different from what we see.

(III) Our ignorance about the initial microstate of $S_{k+1}$ is often illustrated by appealing to some random sampling of a point out of $M_{0}$. Of course, this idea need not be taken too seriously (as describing a fairy tale about some mechanism of selection). However, the point to be stressed here is the following. A random sampling is a sampling that depends only on the measure. The measure with respect to which the sampling is random need only be the measure that fits the observed relative frequencies in experience. In particular the measure need not be the Lebesgue measure, and may not even be conserved under the dynamics. By appealing to the probability measure we can now justify statements about the probability of randomly sampling initial conditions for subsystems of the universe. Here unlike the statement (iii) of the typicality approach, the sampling is described in terms of probability rather than typicality. The role of the measure in our approach is derivative rather than fundamental and is patently probabilistic.

\section{Are There Natural Measures?}

In the literature there are attempts to justify the choice of the measure (in the typicality approach and in other approaches) on the basis of dynamical considerations. An argument sometime given for preferring the Lebesgue measure as 'natural' on the basis of the classical dynamics is the invariance of the Lebesgue measure under the dynamics as expressed by Liouville's theorem. If a measure is invariant under the dynamics it means that the measure of a given set of points in the state space is equal to the measure of the set to which it is mapped by the time evolution equations for all times. Of course this feature has very attractive properties (simplicity, elegance, etc.) but it is unclear why this fact is relevant at all to the issue at stake, namely the explanation and prediction of physical behavior. 
A similar argument is sometimes given in the case of ergodic dynamics. Obviously, the ergodic theorem gives a preferred status to the Lebesgue measure (or to any measure absolutely continuous with the Lebesgue measure) since it shows that the relative frequency of any macrostate $\mathrm{M}$ along an infinite trajectory is equal to the Lebesgue measure of $\mathrm{M}$ for a Lebesgue measure one of points in the phase space of the system. There are various senses in which the preferred status of the Lebesgue measure here is irrelevant for the issue at stake. First, the ergodic theorem yields no predictions concerning finite times, and therefore strictly speaking the theorem is not empirically testable. For example, it is extremely difficult to distinguish empirically between an ergodic system and a system with KAM dynamics (see Earman and Redei 1996). Second, even if the dynamics of the universe is granted to be ergodic and even if one accepts the fairy tale about an initial random sampling, this does not imply that the sampling is random relative to the Lebesgue measure. One can say metaphorically that God could have used a non-Lebesgue sort of die in sampling at random the initial condition of the universe even if the universe were ergodic. Third, and with respect to the typicality approach. Consider again statement (iii) in section 1. Here the idea is that the fact that $\mathrm{T} 1$ has measure (close to) one suggests that the initial condition of the universe belongs to T1. Since the measure is not to be understood as a probability measure, this seems to mean that the measure zero set is excluded as impossible in some sense. But the measure zero set belongs to the initial macrostate of the universe and we don't see what justifies this exclusion.

Finally, it is important to stress in this context that in understanding the ergodic theorem as a theorem about probability one must identify from the outset that a set of Lebesgue measure zero (one) has zero (one) probability. This identification is not part of von Neumann's and Birkhoff's ergodic theorem, although the theorem is usually understood in probabilistic terms. However, whether or not the Lebesgue measure may be interpreted as the right probability measure for thermodynamic systems depends on whether it satisfies our probability rule $\left(^{*}\right)$.

Another argument sometimes given for taking the Lebesgue measure as the natural measure in statistical mechanics is that the Lebesgue measure of a macrostate corresponds to the thermodynamic entropy of that macrostate. However, this 
correspondence is true only if the Second Law of thermodynamics (even in its probabilistic version) is true. But as we argued elsewhere (see our 2010a, 2010b and Albert 2000, Ch. 5) the Second Law of thermodynamics is not universally true in statistical mechanics.

\section{Measure One Theorems in Classical Statistical Mechanics}

The above conclusion has implications for the significance of measure one theorems in statistical mechanics. We focus here as an example on Lanford's theorem. ${ }^{7}$ Lanford proved on the basis of the classical equations of motion, that, roughly, given some specific initial macrostate, and some specific kind of Hamiltonian, a Lebesgue measure one of the microstates in that macrostate will evolve to a macrostate with larger entropy, after a certain short time. ${ }^{8}$ Can such a theorem endow the Lebesgue measure with a status that is stronger than that of an empirical generalization (as sketched in (*) above)?

In terms of our transition probabilities Lanford's theorem proves that the Lebesgue measure of the overlap between the blob $B\left(t_{0}+\Delta t\right)$ and the macrostate $E$ of equilibrium (or some other high entropy macrostate) is 1 . Of course, as we said above, since the Lebesgue measure is conserved under the dynamics, one may interpret Lanford's theorem as referring to the Lebesgue measure of subsets of the initial macrostate $M_{0}$ at $t_{0}$. However, inferring anything about the measure of subsets of the initial macrostate is an artifact of the contingent fact that the Lebesgue measure matches the observed relative frequencies.

Another crucial point in this context is the following. There are two different and logically independent ways of understanding the role of the Lebesgue measure in Lanford's theorem. (A) The size of the overlap between the blob $B\left(t_{0}+\Delta t\right)$ and the macrostate E, as determined by the Lebesgue measure, is 1; (B) Upon a random sampling of a point out of the blob $B\left(t_{0}+\Delta t\right)$, one is highly likely to pick out a point from the overlap of the blob with $E$. The distinction between (A)-type statements about sizes of sets and (B)-type statements about probabilities is general.

\footnotetext{
${ }^{7}$ For details concerning Lanford's theorem see Uffink (2007).

${ }^{8}$ The fact that a Maxwellian Demon is compatible with classical statistical mechanics demonstrates that there can be no theorem in mechanics that implies a universal entropy increase. See Albert (2000, Ch. 5) and Hemmo and Shenker (2010, 2011).
} 
Lanford's theorem is about the size of the overlap with $E$, that is, it is only an (A)type theorem, whereas in order to make predictions about the future behavior of Stype systems (such as our $S_{k+1}$ in the above example) one needs to add a (B)-type statement, which is not proven by Lanford's theorem. In other words, assuming that we already know from experience that the Lebesgue measure of the overlap regions (of the blob with the macrostates) matches the relative frequencies of the macrostates, Lanford's theorem provides possible mechanical conditions, which underwrite these observations.

To appreciate this point, note that if the measure $\mu$ that matches our experience were not the Lebesgue measure, but some other measure (that may not be absolutely continuous with Lebesgue) then Lanford's theorem would have a completely different significance: for instance, it could happen that by the measure $\mu$ the number of systems that go to equilibrium given Lanford's Hamiltonian would be small. The theorem that a set of Lebesgue measure one of points has a certain property (such as approaching equilibrium after some finite time interval) would be empirically insignificant - unless this fact is supplemented by the additional fact that the Lebesgue measure happens to correspond (to a useful approximation) to the observed relative frequencies.

The general structure of Lanford's theorem is that it proves a certain statement about the dynamics of the form of (i) in the typicality approach (see section 2). That is, Lanford's theorem shows that a certain subset of micrsostates $\mathrm{T} 1$ share some property F (entropy increase, for example), such that all the points in $\mathrm{T} 1$ are mapped by the dynamics to points in T1*. Moreover, the theorem shows that the subset T1 has Lebesgue measure one. But nothing in this theorem justifies the choice of the measure. In particular, the fact that T1 has Lebesgue measure one does not constitute such a justification. What's important in Lanford's theorem is that it identifies two sets $\mathrm{T} 1$ and $\mathrm{T} 1 *$ and proves that $\mathrm{T} 1$ evolves to $\mathrm{T} 1 *$ under the dynamics. That is, it is about the structure of trajectories. The fact that $\mathrm{T} 1$ has Lebesgue measure one is important only if there are independent reasons for preferring the Lebesgue measure. As we saw in section 3 such reasons can be grounded essentially only in experience. 


\section{Typicality in Bohmian Mechanics}

The question of typicality has been considered extensively and explicitly not only in classical statistical mechanics but also in the context of Bohm's alternative theory to quantum mechanics by Dürr, Goldstein and Zanghi (1992, hereafter DGZ). DGZ prove that a typical Bohmian trajectory exhibits relative frequencies of measurement outcomes that conform to the quantum mechanical Born rule. Here is what they say about their proof:

“...To demonstrate the compatibility of Bohmian mechanics with the predictions of the quantum formalism, we must show that for at least some choice of initial universal $\Psi$ and $q$, the evolution [given by Bohm's velocity equation] leads to apparently random pattern of events, with empirical distribution given by the quantum formalism. In fact we show much more.

We prove that for every initial $\psi$ this agreement with the predictions of the quantum formalism is obtained for typical - i.e. for the overwhelming majority of - choices of initial $q$. And the sense of typicality here is with respect to the only mathematically natural - because equivariant - candidate at hand, namely, quantum equilibrium.

Thus, on the universal level, the physical significance of quantum equilibrium is as a measure of typicality, and the ultimate justification of the quantum equilibrium hypothesis is, as we shall show, in terms of the statistical behavior arising from a typical initial configuration.” (DGZ 1992, p. 859)

In other words, DGZ argue that for every initial universal wavefunction and for $a$ typical initial global configuration, the probability distribution over the Bohmian position of subsystems of the universe is given by the absolute square of the effective wavefunction of the subsystems (when an effective wavefunction exists). From this result they show that the conditions sufficient for the laws of large numbers to hold are satisfied in Bohmian mechanics with probability distribution that recovers the predictions of standard quantum mechanics as given by Born's rule. Here the notion 
of a typical global configurations is understood relative to the quantum mechanical measure, i.e. the absolute square of the universal wavefunction.

What is the role of the typicality assumption in this argument? Typicality is meant to replace here probability over initial conditions of the universe, and thus explain the initial conditions while avoiding the fairy tale concerning the random or probabilistic choice of initial conditions. As we argued above, there are two problems with this approach. First, if the notion of typicality is non-probabilistic, it is unclear why a condition which is true for most initial conditions (relative to the measure) should be taken as true for a given system. The problem is to justify the choice of the measure of typicality (in this case, the absolute square of the universal wavefunction) in a noncircular way. The argument given by DGZ for preferring the quantum mechanical measure as natural is that it is the only equivariant measure under the dynamics of the wave function and the Bohmian dynamics of the initial $q$. However this argument is irrelevant just as the classical Liouville's theorem is irrelevant as an argument for preferring the Lebesgue measure in the case of ergodic dynamics.

It is important to stress that this criticism concerning the notion of typicality does not in anyway undermine the important results by DGZ concerning the probabilistic content of Bohm's theory. In fact, it seems to us that one can retain all these results by making the simpler assumption that the initial condition of the universe is as a matter of fact of the kind that yields the quantum mechanical predictions. This fact need not be further justified, in particular, it need not (and in our view cannot) be justified by reference to a set of typical initial conditions. We have no empirical access to the initial configuration of the universe and to the universal wavefunction. We construct the set of initial conditions and the wavefunction in a way that yields the probabilistic content of the theory, as it is given by the quantum mechanical Born rule (the absolute square of the (effective) wavefunction of subsystems of the universe in Bohm's theory), where the Born rule itself is subject to empirical tests in our experience. We then find a measure of typicality according to which the right sort of initial universal conditions turn out to be typical. But evidently, such a procedure can be meaningful 
only if by construction the properties in which one is interested are the same for the vast majority of the admissible initial conditions. ${ }^{9}$

\section{Conclusion}

We argued that typicality considerations are not justified and don't have a convincing explanatory value in classical and Bohmian mechanics. These theories, however, are deterministic. The question remains whether the above criticism is applicable in stochastic theories such as standard quantum mechanics (say, in von Neumann's formulation) or the Ghirardi-Rimini-Weber collapse theory. Recall that the motivation for appealing to typicality as we understand it is the desire to dispense with the fairy tale associated with postulating probability distributions over the initial conditions of the universe. In stochastic theories, although the idea of a probability distribution over initial conditions may be relevant in more or less the same sense it has in deterministic theories, it may be that the choice of measure is dictated by the stochastic dynamics. This may have interesting implications for the idea of typicality. We leave this question open for future research.

\section{Acknowledgement}

\section{References}

Albert David (2000) Time and Chance (Harvard University Press).

Callender Craig (2007) The Emergence and Interpretation of Probability in Bohmian Mechanics Studies in History and Philosophy of Modern Physics 38, pp. 351-370. Dürr Detlef, Goldstein Sheldon and Zanghi Nino. (1992) Quantum Equilibrium and the Origin of Absolute Uncertainty, Journal of Statistical Physics 67(5/6), pp. 843907.

Earman John and Miklos Redei (1996) Why Ergodic Theory does not Explain the Success of Equilibrium Statistical Mechanics, The British Journal for the Philosophy of Science, 47, pp. 63-78.

Einstein Albert (1905) Über die von der molekularkinetischen Theorie der Wärme geforderte Bewegung von in ruhenden Flüssigkeiten suspendierten Teilchen, Annalen

\footnotetext{
${ }^{9}$ This last sentence is a paraphrase of a sentence by E. Wigner cited by Goldstein et al. (2010, p. 10).
} 
der Physik 17, pp. 549-560. English translation in: R. Furth (ed.) Albert Einstein, Investigations on the Theory of Brownian Motion (New York, Dover, 1926).

Goldstein Sheldon, Lebowitz Joel, Mastrodonato Christian, Tumulka Ruderich and Zanghi Nino (2010a) Normal Typicality and von Neumann's Quantum Ergodic Theorem, Proceedings of the Royal Society A 466 (2123), pp. 3203-3224

Goldstein Sheldon, Lebowitz Joel, Mastrodonato Christian, Tumulka Ruderich and Zanghi Nino (2010b) Approach to Thermal Equilibrium of Macroscopic Quantum Systems, Physical Review E 81 (011109).

Hemmo Meir and Shenker Orly (2010) Maxwell's Demon, The Journal of Philosophy, Vol. 107, No. 8 (August 2010), pp. 389-411.

Hemmo Meir and Shenker Orly (2011) Szilard's Perpetuum Mobile, Philosophy of Science, Vol. 78, No. 2 (April 2011), pp. 264-283.

Maudlin Tim (2007) What Could Be Objective About Probabilities? Studies in History and Philosophy of Modern Physics 38, pp. 275-291.

Pitowsky Itamar (1992) Why Does Physics Need Mathematics? A Comment, in: E Ulmann-Margalit (Ed.), The Scientific Enterprise (Dordrecht, Kluwer), pp. 163-167. Uffink Jos (2007). Compendium to the Foundations of Classical Statistical Physics, in: J. Butterfield and J. Earman (Eds.), Handbook for the Philosophy of Physics, Part B, pp. 923-1074. 